\title{
Graphite Grains and \\ Graphite-Core-Ice-Mantle Grains
}

\author{
N. C. Wickramasinghe \\ California Institute of Technology \\ Pasadena, California
}

\begin{abstract}
$A^{\mathrm{M}}$ MONG THE FIRST REQUIREMENTS FOR A GRAIN MODEL is that it should explain the observed interstellar extinction law or laws as they are now understood; a desirable feature of such a model is that as few ad hoc assumptions be made as possible.

A criticism of the classical ice grain theory is that a very narrow range of grain sizes (or a size distribution with a size parameter specified to within a few percent) must be postulated in order to obtain a fit with the mean extinction law. (See refs. 1 and 2.) While it is true that regional variations in the extinction law have recently been detected (refs. 3 and 4), the best available evidence indicates that the extinction law is quite uniform when averaged over individual large regions which are widely distributed in the galaxy. (See ref. 5 and paper by Nandy in the present compilation.) The restriction of particle size to a radius within a few percent of an arbitrarily specified value $\left(r \approx 3 \times 10^{-5} \mathrm{~cm}\right)$ demanded on the basis of pure ice absorption is therefore considered quite unsatisfactory, particularly in view of the fact that no characteristic size parameter emerges from the Oort-van de Hulst theory for the formation and destruction of grains. (See ref. 6.)

In the original graphite model for interstellar extinction (refs. 7 and 8) this difficulty was overcome quite simply. In reference 7 , the situation was that the mean extinction law as given in reference 9 was reproduced with graphite particles which were small enough $\left(r<5 \times 10^{-6} \mathrm{~cm}\right)$ for a Rayleigh scattering approximation to the extinction cross section to be valid. This upper limit to the grain size was derived on the basis that there was insufficient time to build bigger grains, for example, in the pulsation cycles of $\mathrm{N}$ stars. Although now the situation for graphite is not as simple as it then seemed, the results of the present study show that one needs only to specify an upper limit to the graphite core radius and have an equilibrium distribution of ice mantles in order to reproduce the observed extinction laws.
\end{abstract}


Another important feature of graphite grains is that they can explain simply the phenomenon of interstellar polarization, as suggested originally by the authors of reference 10 . The reason is that graphite is a strongly anisotropic substance with an electrical conductivity about a hundred times greater in directions parallel to the basal planes than at right angles to them. Because of this feature, graphite crystals have strongly polarizing properties. Furthermore, graphite is diamagnetic, and thus graphite flakes are easy to aline in a galactic magnetic field. In reference 10, however, only a small fraction of all the grains in the line of sight of a star were assumed to be polarizing graphite flakesthe others were taken to be nonpolarizing ice grains which gave rise only to the selective extinction. For such a model the total alinement of all the graphite flakes present was required in order to produce the observed ratio of polarization to extinction $p / A_{v}$; and to accomplish this alinement one needs a galactic magnetic field of about $10^{-5}$ gauss. (See ref. 11.) This value is probably greater than the best available estimates for the galactic magnetic field. If, however, one assumes that graphite particles (or graphite particles with ice mantles) produce the extinction as well as the polarization, the situation is much better. The grains then need to be alined only to a very slight extent - about 2 percent - in order to produce the observed $p / A_{v}$ ratio, and the magnetic field required to produce such alinement is less than $10^{-6}$ gauss. (See refs. 12 and 13.) Magnetic fields of this general order are now considered far more satisfactory than the fields of $10^{-4}$ to $10^{-5}$ gauss which are required to aline "dirty ice" grains. (See ref. 14.)

\section{MIE COMPU'TATIONS FOR GRAPHITE}

At the time of the original extinction computations for graphite grains (refs. 7 and 8), the available optical data on graphite were still very limited. The complex refractive index in directions parallel to the basal planes was given by (ref. 7 )

$$
m^{2}=K-(2 \mathrm{i} \sigma \lambda) / c,
$$

where $c$ is the velocity of light. The dielectric constant $K$ and the optical conductivity $\sigma$ had to be specified at a given wavelength $\lambda$. The following values were suggested on the basis that they were consistent with earlier data on the reflectivities of polished graphite surfaces:

$$
\left.\begin{array}{l}
\sigma \cong 1.2 \times 10^{15} / \mathrm{sec} \\
K \cong 2
\end{array}\right\}
$$

These values used in equation (l) yielded

$$
m^{2}=2-8 i \lambda \text {, }
$$

where $\lambda$ is the wavelength expressed in microns. 
Mie computations performed on the basis of this model refractive index in references 8 and 15 substantiated the general conclusion, already reached in reference 7 by a simple Rayleigh approximation, that the extinction law as given in reference 9 could easily be reproduced for very small graphite particles - provided the radii are all less than about $5 \times 10^{-6} \mathrm{~cm}$. The assumption of a wavelength-independent conductivity for graphite, however, was rather unsatisfactory, although it was the best possible at the time. (See ref. 16.) These original Mie computations were, therefore, subject to revision when better data became available.

The first direct measurements of the wavelength dependence of the refractive index of graphite single crystals were made by the authors of reference 17, who measured the absorptive index $\kappa$ in the wavelength range $2100 \AA<\lambda<5500 \AA$. Their results showed a sharp peak in $\kappa$ near $2600 \AA$, typical of a resonance phenomenon, which could not be explained in terms of a constant conductivity. This absorption peak centered at $2600 \AA$ arises because of a transition of $\pi$-electrons into the conduction band, the precise position of the peak corresponding to the energy difference $(4.8 \mathrm{eV})$ between the state of maximum density in the conduction band and the adjacent valence band of graphite. Such an effect was, in fact, predicted theoretically for graphite in reference 18 .

The qualitative features of the measurements of reference 17 seemed to be consistent with a model of the refractive index based on the Drude-Lorentz theory of anomalous dispersion. (See ref. 19.) This model was then used to extrapolate to values of $\lambda$ outside the measured range $2100 \AA<\lambda<5500 \AA$, and Mie computations were performed. The extinction curves for very small particles showed a steep rise toward 2200

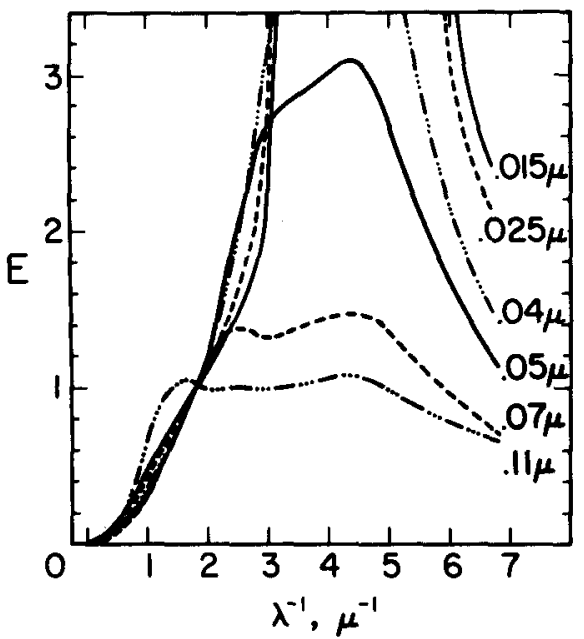

Figure 1. - Extinction curves for graphite spheres of various radii. Normalization: $E=Q_{e x t}(\lambda) / Q_{e x t}(5470 \AA)$.

271-992 O-67-12 
$\AA$, exhibiting the qualitative features of the rocket UV observations (refs. 20 and 21).

More recently, however, direct measurements of both the real and imaginary parts of $m^{2}$ have been made (refs. 22 and 23) over wavelengths ranging from the far infrared to the far ultraviolet. There is little doubt that these measurements represent the best available optical data on graphite, and rigorous Mie computations have been performed for this case as well (refs. 1 and 24). Normalized extinction curves defined by

$$
E=Q_{e x t}(\lambda) / Q_{e x t}(5470 \AA)
$$

for various particle radii $\left(Q_{e x t}\right.$ is the extinction efficiency), are plotted in figure 1. (See also refs. 1 and 24.) For particle radii less than about 0.06 $\mu$, the extinction curves follow an approximately $\lambda^{-1}$ dependence in the wavelength range $0.8 \mu^{-1}<\lambda^{-1}<2.4 \mu^{-1}$ and show considerable variation for shorter wavelengths. The precise shape of the ultraviolet portion of the curve depends on the particle radius, but small particles produce a sharp ultraviolet quenching effect centered at $\sim 2200 \AA$. This feature may be of importance in explaining the recent ultraviolet observations

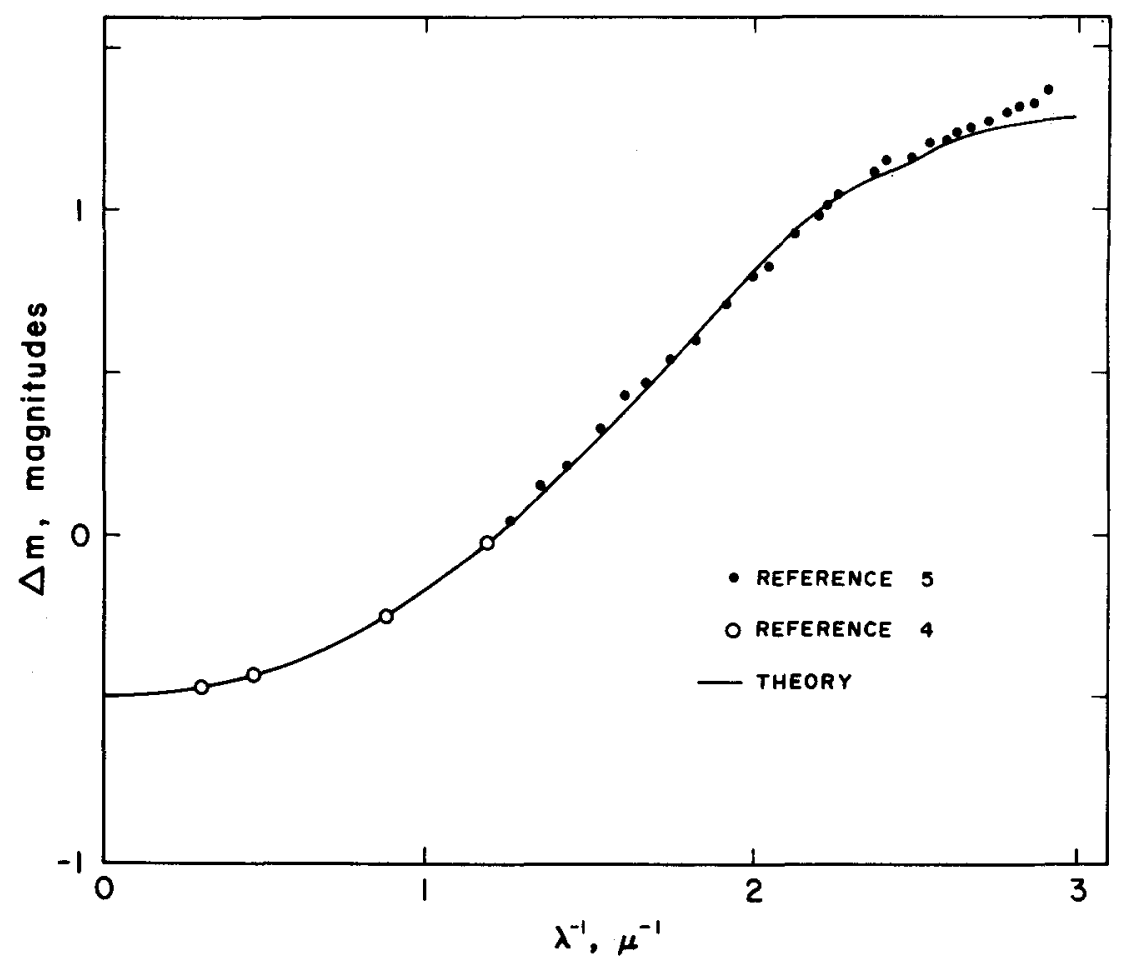

FIGURE 2. - Comparison of theoretical curve for a gaussian distribution of graphite spheres centered at $0.55 \mu$ with dispersion $0.01 \mu$ with observational points for Perseus region. Normalization to $\Delta m=0$ at $\lambda^{-1}=1.22, \Delta m=1$ at $\lambda^{-1}=2.22$. 
presented in references 20 and 21 . It is further of interest that the characteristic wavelength $\lambda^{-1}=2.4 \mu^{-1}$ obtained by Nandy (see refs. 3 and 25 and paper by Nandy in the present compilation), at which both the Cygnus and Perseus extinction laws deviate from a $\lambda^{-1}$ dependence, also turns out to be the wavelength at which extinction curves for small graphite spheres begin to deviate markedly as a function of $\lambda^{-1}$, as can be seen in figure 1 .

The situation then is that the recent ultraviolet observations of a peak in extinction at $2200 \AA$ and also a knee at $\lambda^{-1}=2.4 \mu^{-1}$ in the curves of references 3 and 25 emerge as properties of the recently measured refractive index of graphite.

Detailed fits have been obtained on the basis of graphite grains and graphite grains covered with ice for the best available observational extinction curves. (See ref. 5.) In figure 2 is given a comparison of the Perseus curve of references 4 and 5 with the theoretical curve for a Gaussian distribution of graphite grains with dispersion $\sigma=0.01 \mu$ centered at $0.055 \mu$.

\section{ICE MANTLES}

The premise that graphite grains ejected from stars tend to grow icelike mantles in interstellar clouds is presented in references 2,8 , and 26 . A simple model for such growth can be constructed on the assumption that a certain fraction $\alpha$ of heavy atoms such as $\mathrm{O}, \mathrm{C}$, or $\mathrm{N}$ which hit a grain stick on as "icelike"' molecules. The rate of increase of radius $r$ is then given by

$$
\frac{\mathrm{d} r}{\mathrm{~d} t}=\frac{\alpha n}{s}\left(\frac{k T m}{2 \pi}\right)^{1 / 2}
$$

where

$n$ number density of $\mathrm{O}, \mathrm{C}$, or $\mathrm{N}$, in ambient gas

$m$ mean atomic weight of heavy atoms

$\alpha$ sticking probability

$T$ kinetic temperature of gas

$s$ mean density of grain material

$k$ Boltzmann constant

Taking $m \cong 18, s \cong 1.1 \mathrm{~g} / \mathrm{cm}^{3}, T \cong 100^{\circ} \mathrm{K}, n_{\mathrm{H}} \cong 10 / \mathrm{cm}^{3}$ and $n / n_{\mathrm{H}} \cong 10^{-3}$ as representative values for a cloud yields

$$
\frac{\mathrm{d} r}{\mathrm{~d} t} \cong 7 \alpha \times 10^{-14} \mathrm{~cm} / \mathrm{yr}
$$

If $\alpha \cong 1$, as is commonly supposed, a graphite grain of initial radius $3 \times 10^{-6} \mathrm{~cm}$ will grow an ice mantle to about $3 \times 10^{-5} \mathrm{~cm}$ in a few times $10^{8}$ years. The light scattering properties for such a grain will be almost 
the same as those for a pure ice grain of radius $3 \times 10^{-5} \mathrm{~cm}$, except perhaps in the far infrared where core absorption may become dominant. Although it is possible that such icelike grains may be able to grow from graphite nuclei in localized regions, in general it is unlikely. According to reference 26, the proportion of grains with large mantles in the line of sight of the star is likely to be very small because of the disruptive effect of cloud collisions and because of the limited supply of $\mathrm{O}$ atoms which are responsible. These effects are discussed in the following paragraphs.

\section{Mantle destruction by sputtering}

On the average, an interstellar cloud is involved in a collision with a similar cloud once every $10^{7}$ years. If such collisions are adequately inelastic, a cloud may be heated to $3000^{\circ}$ to $4000^{\circ} \mathrm{K}$ and remain at that temperature for a length of time determined by the cooling rate. The best estimates of the cooling rate give a time scale

$$
t \cong 10^{4} / n_{\mathrm{H}_{2}} \text { yr }
$$

where $n_{\mathrm{H}_{2}}$ is the number density of $\mathrm{H}_{2}$ in the colliding clouds. (See ref. 26.) If $t>10^{6}$ years, the available data relating to the sputtering of ice by $\mathrm{H}$ impacts (ref. 26) indicate that all the ice mantles present may be destroyed. However, the condition $n_{\mathrm{H}_{2}}<10^{-2} / \mathrm{cm}^{3}$ required for this does not seem likèly to be realized for collisions between $H I$ clouds, but would be realized for cloud collisions following closely upon cloud encounters with $O$ and $B$ stars. The other type of collision (collisions between $\mathrm{H}$ II clouds) has been estimated to occur with a frequency of once every $10^{8}$ years per cloud, so that the chance of random destruction of an ice mantle is

$$
P=10^{-8} / \mathrm{yr}
$$

Graphite cores are not destroyed by this process.

\section{A possible exhaustion of oxygen}

A further restriction on the sizes of ice mantles might arise because of a limit of the available $O$ atoms relative to the mass of condensed graphite cores. Graphite cores appear to be indestructible by ordinary interstellar processes, while $O$ atoms tend to flit to and fro between manthes and gas. The characteristic time scale for the release of an $\mathbf{O}$ atom from a mantle is about $10^{8}$ years; this is also the time scale for the growth of an ice mantle up to a radius of about $10^{-5} \mathrm{~cm}$. An equilibrium in

$$
\mathrm{O}_{\text {grains }} \rightleftarrows \mathrm{O}_{\text {gas }}
$$


is therefore likely to be reached during the age of the galaxy in which

The process

$$
n\left[\mathrm{O}_{\text {grains }}\right] \approx n\left[\mathrm{O}_{\text {gas }}\right]
$$

$$
\mathrm{C}_{\mathrm{gas}} \rightarrow \text { Stars } \rightarrow \mathrm{C}_{\text {grains }}
$$

on the other hand, is likely to proceed predominantly one way, and an appreciable fraction of the available interstellar $\mathrm{C}$ atoms may eventually become locked up in grains. If this happens, the size of a typical ice mantle can be computed. Assuming an interstellar composition similar to the solar case,

$$
\frac{\text { Mass of ice mantles }}{\text { Mass of C cores }} \cong \frac{18 \times 2.9 \times 10^{7}}{12 \times 1.7 \times 10^{7}} \cong 2.6
$$

For graphite with a mean core radius of $3 \times 10^{-6} \mathrm{~cm}$ and a density of $2.5 \mathrm{~g} / \mathrm{cm}^{3}$, a typical mantle can grow to at most $x \times 3 \times 10^{-6} \mathrm{~cm}$, where

$$
\begin{aligned}
\left(\frac{1}{2.5}\right)\left(\frac{x^{3}-1}{1}\right) & \cong 2.6 \\
x & \cong 2
\end{aligned}
$$

A typical graphite core of radius $3 \times 10^{-6} \mathrm{~cm}$ is therefore able to grow to only about $6 \times 10^{-6} \mathrm{~cm}$ with ice.

\section{Equilibrium size distribution of mantles}

On the assumption that the processes of mantle growth and destruction have reached an equilibrium state, one can obtain an equilibrium size distribution of ice mantles and perform extinction computations for such a distribution.

First suppose that the graphite cores are all of one radius $r_{0}$. Let $n(r, t) \mathrm{d} r, r \geqslant r_{0}$ be the number of grains with mantle radii in the range $r, r+\mathrm{d} r$ at time $t$. The total number of grains with mantles of radius less than $r$ is then

$$
N(r, t)=\int_{r_{0}}^{r} n(r, t) \mathrm{d} t
$$

Denoting by $P$ the destruction probability of a mantle per unit time, the conservation of cores gives

$$
\begin{gathered}
N(r+\mathrm{d} r, t+\mathrm{d} t)=N(r, t)-P \mathrm{~d} t N(r, t) \\
\frac{\partial N}{\partial r} \mathrm{~d} r+\frac{\partial N}{\partial t} \mathrm{~d} t=-P N \mathrm{~d} t
\end{gathered}
$$

In a steady state $\partial N / \partial t=0$, so that 


$$
\begin{gathered}
\frac{\partial N}{\partial r} \dot{r}=-P N \\
n(r) \dot{r}=-P \int_{r_{0}}^{r} n(r) \mathrm{d} r \\
\frac{1}{n} \frac{\mathrm{d} n(r)}{\mathrm{d} r}=-P / \mu
\end{gathered}
$$

where $\mu=\mathrm{d} r \equiv \dot{r}$, the rate of growth of grain. Thus

$$
n(r)=n\left(r_{0}\right) \exp \left[-\frac{P}{\mu}\left(r-r_{0}\right)\right]
$$

For a given value of $r_{0}$ and a known value of the ratio $P / \mu$ (determined from equations (6) and (8)), equation (10) gives the equilibrium size distribution of ice mantles. However, both these estimates are uncertain to within factors of order unity, and the ratio $P / \mu$ can therefore also be specified only to within a factor of order unity.

Two representative cases are considered:

(1) $P / \mu=14.28 \mu^{-1}$

(2) $P / \mu=33.33 \mu^{-1}$

Case (1) corresponds to $P=10^{-8} / \mathrm{yr}$ and $\mu=7 \times 10^{-14} \mathrm{~cm} / \mathrm{yr}$, and case (2) corresponds to $P=2 \times 33 \times 10^{-7} / \mathrm{yr}$ and $\mu=7 \times 10^{-14} \mathrm{~cm} / \mathrm{yr}$; but only the ratio $P / \mu$ is relevant for determining the size distribution. The functions $n(r) / n\left(r_{0}\right)$ for these two cases are plotted in figure 3 .

Rigorous extinction computations are given in reference 27 for these two size distributions of mantles. The computations were initially made for a single value of the core radius $r_{0}=0.03 \mu$, but it was subsequently verified that the restlting normalized extinction curves drawn in figure 4 were insensitive to the precise value of the core radius, provided $r_{0}<\sim 0.06 \mu$. Therefore, one only has to impose an upper limit $r_{0}<0.06 \mu$ on the sizes of graphite cores to produce the normalized curves plotted in figure 4. It turns out that curve (2) (fig. 4), corresponding to $P / \mu=33.33$ $\mu^{-1}$, reproduces the Cygnus law given in reference 25, and it also qualitatively gives the observed ultraviolet absorption peak. (See ref. 21.) The detailed agreement with the observational points of reference 21 is shown in figure 5 . The agreement is seen to be very good. In the near ultraviolet, the agreement can further be improved by adjusting the value of $P / \mu$; but the theoretical curve already passes through the root-mean-square dispersion of the near $\mathrm{UV}$ points among the large number of stars observed by the author of reference 25 .

Another question of interest concerning graphite-core-ice-mantle grains is: What fraction of the total extinction from a given graphite- 


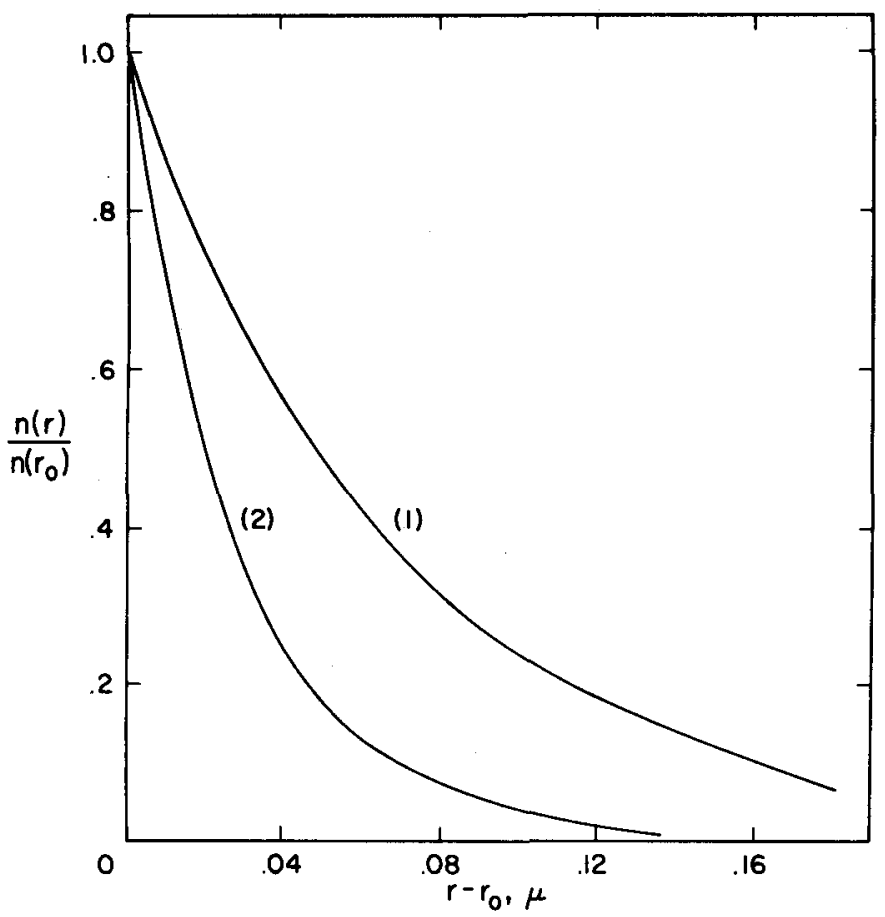

FIGURE 3. - Equilibrinm distribution function for mantle radii $n(r) / n\left(r_{0}\right)$ for the two cases (1) $P / \mu=14.28$ micron $^{-1}$, and (2) $P / \mu=33.33$ micron $^{-1}$.

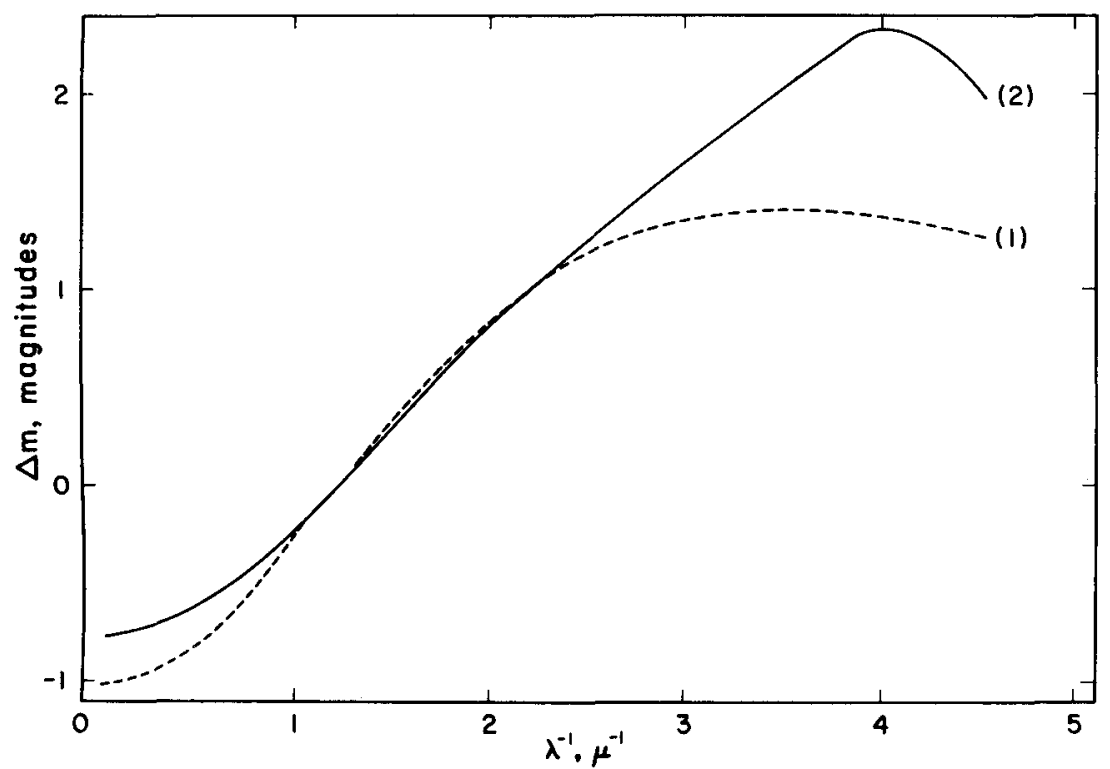

FigURE 4. - Normalized extinction curves for the two ice. mantle size distributions sketched in figure 3, for graphite cores with $r_{0}<\sim 0.06 \mu$. Normalization to $\Delta m=0$ at $\lambda^{-1}=1.22$; $\Delta m=1$ at $\lambda^{-1}=2.22$. $\mu=7 \times 10^{-14} \mathrm{~cm} / \mathrm{yr}$; curve (1): $P=10^{-8} / \mathrm{yr}$; curve (2): $P=2.33$ $\times 10^{-7} / \mathrm{yr}$. 


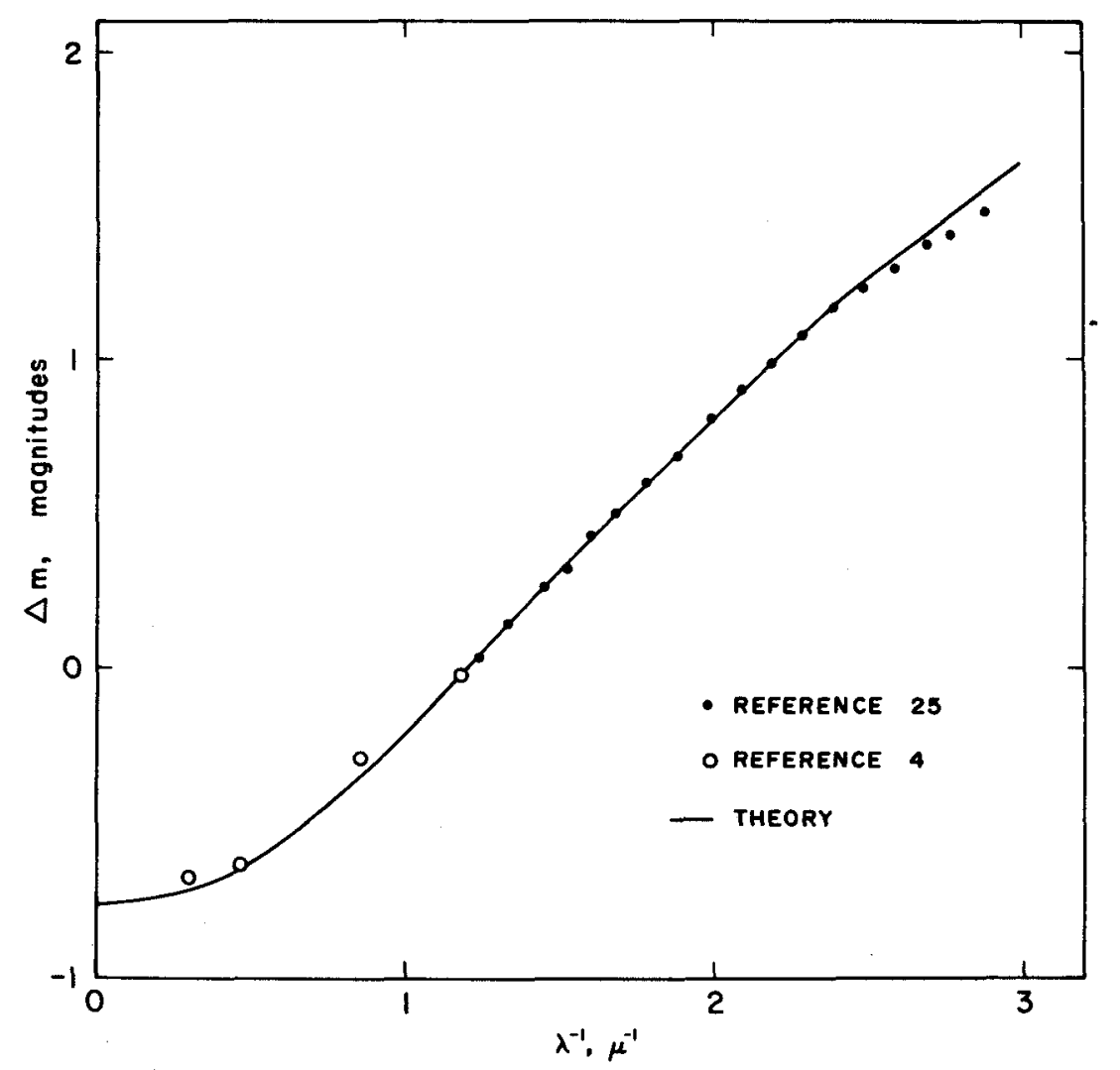

FIGURE 5. - Comparison of theoretical curve for equilibrium distribution (curve for case (2) in fig. 3) with observational points for Cygnus region. Normalization to $\Delta m=0$ at $\lambda^{-1}$ $=1.22 ; \Delta m=1$ at $\lambda^{-1}=2.22$.

core-ice-mantle grain arises from core absorption? Figure 6 presents the total extinction cross sections for a graphite core of radius $r_{0}=0.02 \mu$ covered to varying extents with ice. It is seen that for such a core covered with ice up to twice its own radius $\left(D \equiv r / r_{0}=2\right)$, the extinction in the infrared and visible comes mainly from absorption in the graphite core. At about $6000 \AA$, in the red, graphite core absorption makes up about 70 percent of the total cross section (comparing ordinates with $D=1$ case), while in the blue and ultraviolet, it contributes to about 45 to 50 percent of the total.

These considerations have a direct bearing on recent infrared observations made in reference 28 as a result of sending up rocketborne equipment designed to detect a strong absorption band at $3.1 \mu$; such a strong absorption band at $3.1 \mu$ is characteristic of ice and is expected on the basis of ice absorption. The fact that such a band was not detected led to the conclusion that ice absorption cannot account for more than 25 


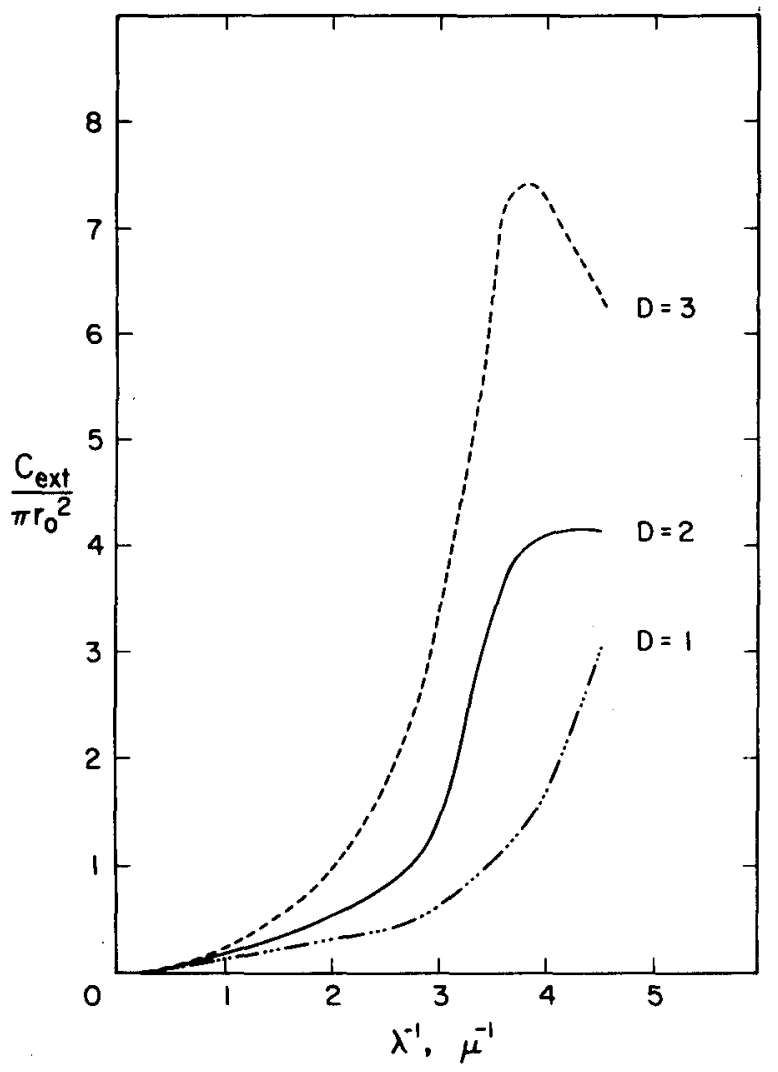

FIGURE 6. - Extinction cross sections for graphite cores with radii $r_{0}=0.02 \mu$ covered with ice up to $D \equiv r / r_{0}=2$ and $D=3 . D=1$ corresponds to pure graphite core.

percent of the total absorption at the visible wavelengths. Although absorption by pure graphite grains would meet this requirement, it is also of interest that the graphite-core-ice-mantle grains considered previously are not incompatible with the observations of reference 28 .

For the Mie computations of the present investigation, spherical particles possessing an isotropic refraction index $m$ have been assumed. The value of the refractive index that was adopted is appropriate for light polarized with an electric vector parallel to the basal planes. Since the absorption cross section for light with an electric vector at right angles to the basal planes is likely to be considerably less than that for light with an electric vector parallel to the basal planes, the present model of spherical isotropic grains is likely to give a good representation for actual graphite flakes provided they are in nearly random orientation. This conclusion, which has been checked for very small anisotropic graphite spheroids by using a Gans approximation (ref. 13), is likely to hold for grains with dimensions less than a few times $10^{-6} \mathrm{~cm}$. 


\section{FORMATION OF GRAPHITE PARTICLES}

In reference 7 it was suggested that graphite particles are formed during the pulsation cycles of $\mathrm{N}$ stars and ejected into interstellar space by the radiation pressure. Among the difficulties which had to be overcome before making graphite particles was the possibility that 0 might compete severely for the $\mathrm{C}$ to form $\mathrm{CO}$. Consider the following thermochemical data:

$$
\begin{aligned}
& \mathrm{C}+\mathrm{O} \rightarrow \mathrm{CO}+11.2 \mathrm{eV} \\
& \mathrm{O}+\mathrm{O} \rightarrow \mathrm{O}_{2}+5.25 \mathrm{eV}
\end{aligned}
$$

Since $\mathrm{CO}$ is more strongly bound than $\mathrm{O}_{2}$, it is likely that the formation of CO may prevent the formation of polyatomic carbon molecules and of graphite at $2000^{\circ} \mathrm{K}$, if there is an excess of oxygen over carbon. However, if there is an excess of carbon over oxygen, as is likely to be true in carbon stars, this difficulty would not arise and solid graphite would be able to condense. The size of grains is limited by the time scale available for condensation during the pulsation cycle of an $\mathrm{N}$ star. It turns out that the maximum permitted radius is about $5.10^{-6} \mathrm{~cm}$, close to the limit essential for the extinction curves.

The conditions for nucleation and growth appear to be considerably more favorable for graphite grains than for ice grains. For ice grains, one is in a better position to redo the calculations of references 29 and 30 using more reliable data. Preliminary calculations indicate that the nucleation problem for icelike grains in interstellar space now looks quite hopeless. The reason is that one cannot form stable diatomic and triatomic molecules at an adequate rate in interstellar space. Even if one formed such molecules in stars, the physical processes that could lead to their growth to "dirty ice" grains are still obscure.

Although the $\mathrm{N}$ stars are probably barely adequate to provide the necessary graphite grain density (ref. 7), it is also worth exploring other possible sites where such grains may be able to form. Preliminary computations which I have made in collaboration with B. Donn and T. P. Stecher indicate that graphite grains may be able to form in the expanding envelopes of certain red giant stars. Even though $n(O) / n(C)=1$ in such envelopes, the lower temperature $\left(\sim 1000^{\circ} \mathrm{K}\right)$ permits a certain fraction of the available $C$ atoms to be stable in the form of bulk graphite.

\section{ALBEDO}

Another requirement usually demanded of interstellar grains is that they. possess a high visual albedo. Although the condition $\gamma \geqslant 0.5$ is frequently quoted, $\overline{\text { it }}$ is difficult to estimate how strong a requirement this must be. Graphite grains of radius of about $0.05 \mu$ have an albedo $\gamma \sim 0.3$ at $5000 \AA$, and smaller graphite grains have lower albedos.

The presence of ice mantles, however, has the effect of considerably 
enhancing the visual albedo, as discussed in reference 27. In table I the albedos of graphite-core-ice-mantle grains with core radii $r_{0}=0.03 \mu$ and $r_{0}=0.05 \mu$ are given. There is clearly no difficulty in meeting the conventional albedo requirement with graphite-core-ice-mantle grains.

\section{TABLE I. - Albedo at Representative Wavelengths}

\begin{tabular}{|c|c|c|c|}
\hline \multirow{2}{*}{$\lambda^{-1}, \mu^{-1}$} & \multicolumn{3}{|c|}{ Albedo for $r / r_{0}=$} \\
\hline & 1 & 2 & 3 \\
\hline \multicolumn{4}{|c|}{$r_{0}($ core $)=0.03 \mu$} \\
\hline 1.56 & 0.04 & 0.17 & 0.51 \\
\hline 1.83 & .06 & .23 & .59 \\
\hline 2.30 & $.11^{\circ}$ & .34 & .67 \\
\hline 3.01 & .19 & .43 & .68 \\
\hline \multicolumn{4}{|c|}{$r_{0}(\operatorname{core})=0.05 \mu$} \\
\hline 1.56 & 0.14 & 0.40 & 0.69 \\
\hline 1.83 & .20 & .47 & .71 \\
\hline 2.30 & .30 & .53 & .74 \\
\hline 3.01 & .42 & .52 & .80 \\
\hline
\end{tabular}

\section{REFERENCES}

1. Wickramasinghe, N. C.; and Guillaume, C.: Interstellar Extinction by Graphite Grains. Nature, vol. 207, 1965, p. 366.

2. Van De Hulst, H. C.: Light Scattering by Interstellar Grains. Pub. Roy. Obs. Edinburgh, vol. 4, 1964, p. 13.

3. NANDY, K.: Observations of Interstellar Reddening II. Results for Region in Perseus. Pub. Roy. Obs. Edinburgh, vol. 5, 1965.

4. Johnson, H. L.: Interstellar Extinction in the Galaxy. Astrophys. J., vol. 141, 1965, p. 923.

5. NANDY, K.; and WICKRAMASINGHE, N. C.: A Survey of Recent Interstellar Reddening Observations. Pub. Roy. Obs. Edinburgh, vol. 5, 1965.

6. OORT, J. H.; and van DE Hulst, H. C.: Gas and Smoke in Interstellar Space. Bull. Astron. Inst., Netherlands, vol. 10, 1946, p. 187.

7. Hoyle, F.; and Wickamasinghe, N. C.: On Graphite Particles as Interstellar Grains. Roy. Astron. Soc., Monthly Notices, vol. 124, 1962, p. 417.

8. Wickramasinghe, N. C.: On Graphite Particles as Interstellar Grains II. Roy. Astron. Soc., Monthly Notices, vol. 126, 1963 , p. 99.

9. Whitford, A. E.: The Law of Interstellar Reddening. Astron. J., vol. 63, 1958, p. 201. 
10. CaYrel, R.; and Schatzman, E.: Sur la Polarisation Interstellaire par des Particules des Graphite. Ann. Astrophys., vol. 17, 1954, p. 555.

11. Cugnon, P.: Sur la Polarisation de la Lumière des Etoiles. Bull. Soc. Royale d. Sciences Liège, vol. 32,1963, p. 228.

12. Wickramasinghe, N. C.: A Note on Interstellar Polarization by Graphite Flakes. Roy. Astron. Soc., Monthly Notices, vol. 125, 1962, p. 87.

13. Wickramasinghe, N. C.: Ph.D. thesis, Cambridge, 1963.

14. Davis, L.; and Greenstein, J. L.: Polarization of Starlight by Aligned Dust Grains. Astrophys. J., vol. 114, 1951, p. 206.

15. Margerum, E. A.; and VAND, V.: Light Scattering by Small Graphite Spheres. Roy. Astron. Soc., Monthly Notices, vol. 128, 1964, p. 431.

16. Hoyl.e, F.; and Wickramasinghe, N. C.: On the Deficiency in the Ultraviolet Fluxes from Early Type Stars. Roy, Astron. Soc., Monthly Notices, vol. 126, 1963, p. 401.

17. Ergun, A.; and McCartney, J. T.: Proceedings of the Fifth Conference on Carbon, vol. 2, Pergamon Press, 1963, p. 167.

18. Coulson, C. A.; and TAYLOR, R.: Studies in Graphite and Related Compounds I Electronic Band Structure in Graphite. Proceedings of the Roy. Soc. London, vol. A65, 1952, p. 815.

19. Wickramasinghe, N. C.: On the Growth and Destruction of Ice Mantles on Interstellar Graphite Grains. Roy. Astron. Soc., Monthly Notices, vol. 131, 1965, pp. 177-190.

20. BogGess, A.; and Borgman, J.: Interstellar Extinction in the Middle Ultraviolet. Astrophys. J., vol. 140, 1964, p. 1636.

21. STECHER, T. P.: Proceedings of IAU Colloquium on Grains. 1965.

22. Taft, E. A.; and PhilipP, H. R.: Optical Properties of Graphite. Phys. Rev., vol. 138A, 1965, p. 197.

23. Carter, J. G.; Huebner, R. H.; Hamm, R. N.; and Birkhoff, R. D.: Optical Properties of Graphite in the Region 1100 to $3000 \AA$. Phys. Rev., vol. 137A, 1965, p. 639.

24. Grevesse-Gulllaume, C.; and Wickramasinghe, N. C.: On the Optics of Small Graphite Spheres. III. Roy. Astron. Soc., Monthly Notices, vol. 132, 1966, pp. 471-473.

25. NANDY, K.: Observations of Interstellar Reddening I. Results for Region in Cygnus. Pub. Roy. Obs. Edinburgh, vol. 3, no. 6, 1964.

26. Wickramasinghe, N. C.: On the Optics of Small Graphite Spheres. I. Roy. Astron. Soc., Monthly Notices, vol. 131, 1966, pp. 263-269.

27. Wickramasinghe, N. C.; Dharmawardhana, M. W. C.; and Wyld, C.: Light Scattering by Graphite Core-Ice Mantle Grains. Roy. Astron. Soc., vol. 134, 1966, pp. 25-36.

28. Danielson, R. E.; Woolf, N. J.; and Gaustad, J. E.: A Search for Interstellar Ice Absorption in the Infrared Spectrum of $\mu$ Cephei. Astrophys. J., vol. 141, 1965, p. 116.

29. Ter HaAr, D.: On the Origin of Smoke Particles in the Interstellar Gas. Bull. Astron. Inst. Netherlands, vol. 10, no. 1,1943, p. 361 .

30. Kramers, H. A.; and Ter HaAR, D.: Condensation in Interstellar Space. Bull. Astron. Inst. Netherlands, vol. 10, no. 137, 1946, p. 371.

\section{DISCUSSION}

Dressler: You have shown that oxygen may not necessarily compete for the carbon in the stellar envelopes you have considered. Could you comment on the effect of hydrogen in the formation of graphite? In others words, can you show that the hydrogen also does not compete with the carbon?

Wickramasinghe: I do not know about hydrogen. The binding energy of $\mathrm{CH}$ is 3.5 electron volts, and the binding energy of $\mathrm{H}_{2}$ is about 4.5 
electron volts. Thus, about $1 \mathrm{eV}$ more strongly bound than $\mathrm{CH}$. For this reason I thought the $\mathrm{H}_{2}$ molecule would begin to form before the $\mathrm{CH}$ molecule, but with the high hydrogen abundance in the stars one cannot be sure unless one performs equilibrium calculations for a $\mathrm{CH}$ system. I am hoping to study this.

Donn: Chemical equilibrium depends upon other factors besides the binding energy. A few years ago Dr. Bauer of Cornell made a very extensive calculation of the equilibrium of the $\mathrm{CH}$ system at high temperatures - temperatures of the order of $1500^{\circ}$ to $5000^{\circ} \mathrm{K}$. One of his results was an estimate of the temperature at which carbon would condense as a function of the $\mathrm{C} / \mathrm{H}$ ratio and the total pressure. Dr. Bauer considered $\mathrm{C} / \mathrm{H}$ ratios as low as $1 / 10$ and pressures from $1 / 10$ atmosphere. Extrapolation of their results to the case which Hoyle and Wickramasinghe discussed for a carbon star yields a condensation temperature of about $2100^{\circ} \mathrm{K}$. This I think is at the minimum of the cycle of variation; therefore, there might be doubt as to whether grains would really grow. In view of the importance of the very suggestive calculations on carbon, it seems desirable to try to make some measurements on the extinction of small carbon grains. One could clarify the problem that Wickramasinghe pointed out about the anisotropy of carbon by measuring the extinction on both carbon black particles, which are more or less spherical poly. crystalline material, and graphite flakes. We have begun some preliminary experiments to investigate this problem.

One can also, I think, modify this experiment to include the effect of an ice mantle by coating some of these particles. Although I am very dubious about getting particles being coated by ice in interstellar space, I certainly think it would be of interest to try to actually make these measurements.

Field: In your first curves, where you are considering graphite alone, there were extraordinary effects in the extreme UV which would not permit a fit to the $\lambda=2200 \AA$ and $\lambda=2600 \AA$ extinctions. Do you think that ice mantles form the only explanation for these data?

Wickramasinghe: No. If one ignored the farthest infrared points of Johnson, one could get a fit all the way up to the points of Boggess and Borgman. One has to take a size distribution of pure graphite to do this. It is to obtain a fit in the far infrared that one needs to coat the graphite particles.

Field: You have a number of curves; some of them seem to go too high and some go too low.

Wickramasinghe: To produce a good fit, or to go through both UV points, either a particular size or a particular size parameter in a size distribution is required. But all the curves for single sizes have the property of possessing peaks centered at $2200 \AA$.

Voice: I think that unity is a very optimistic assumption for the value 
of the sticking coefficient, especially in the early stages of growth before the ice on the surface of the graphite has been nucleated. There is a nucleation problem here, in that graphite is not a very good nucleating agent for this absorption of water.

Wickramasinghe: Yes, the question of whether or not the accommodation coefficient is small is in doubt, and if it is small, not much ice will form. I think there is a very recent paper by Krishna Swamy in which he has a larger size parameter in an Oort-van de Hulst distribution of ice grains which fits the Orion curve very well.

O'Dell: The same result can be obtained if the small particles are removed. But what you say now seems inconsistent with what you said about sputtering, because if grain destruction can occur, then the situation in Orion would be ideal for this process and the grains would tend to be smaller rather than larger than average.

Wickramasinghe: For the Orion nebula, it would depend on the available time scale. I think it requires from $10^{4}$ to $10^{5}$ years to sputter the ice mantle if the gas temperatures are about $10000^{\circ} \mathrm{K}$. What would you say is the age of Orion?

O'Dell: I would say less than $2 \times 10^{6}$ years.

Wickramasinghe: The mantle destruction time would certainly be less than $2 \times 10^{6}$ years.

O'Dell: The fact that you are seeing scattered light in the other H II regions indicates that this grain destruction mechanism is not extremely important. Orion may be different. Now, does this mean that the remaining particles are graphite?

Wickramasinghe: The graphite particles wouldn't be sputtered. It would require a long time to sputter them if the gas temperature were $10000^{\circ} \mathrm{K}$. But with ice I imagine you would get the whole thing sputtered in $10^{5}$ years.

O'Dell: By the same token I wonder whether Orion is more important or more suitable for sputtering than these others?

Wickramasinghe: Isn't the gas density also fairly high in Orion? 'Therefore, one really has to take a balance between the condensation as well as the sputtering. I don't know.

Greenberg: Did I miss a point in understanding the sputtering process? The relative fraction of condensing atoms and of atoms lost by sputtering is a function of the velocity distribution only. If the sputtering process is more effective than the condensing process at low density, the rate of loss by sputtering will certainly be greater as the density is increased.

Wickramasinghe: Yes, this is probably true.

Stecher: I would like to point out, as shown in the graph, that for larger particles the extinction does become neutral (wavelength independ- 
ent). Thus, as you get about three times the size that is necessary to fit Perseus, even in the Oort-van de Hulst distribution, you might find at $\lambda^{-1}=3$ that the extinction is decreasing slightly in the ultraviolet.

Wickramasinghe: Yes. I think one could get a better fit in the ultraviolet by adding small particles unless one insists upon a very close fit in the infrared. By a very close fit $I$ mean a fit through all the circles that Johnson and Borgman have. We did this using pure graphite distributions for Perseus and Cygnus, and the fits are as good as the ones for grains that have mantles.

Stecher: I should think that there would be some justification for adding the effects of several different size distributions to make the theory fit all the features completely. The basis for this justification is that each cloud is different and each region in a cloud is probably different.

Wickramasinghe: With regard to the graphite formation process, Dr. Donn mentioned earlier about the importance of $\mathrm{CH}$ formation. Perhaps a temperature below $2100^{\circ} \mathrm{K}$ is required in order for graphite grain formation to occur. Temperatures as low as this are available in the $\mathrm{N}$ stars. Also, it does seem that several of the carbon stars have opaque atmospheres in the infrared and some emit material similar to that emitted in $\mathbf{R}$ Corona Borealis.

Donn: There is a question in this respect of whether, in $R$ Corona Borealis stars, the variation is due to some condensation process; that is, whether the catastrophic drop of these stars may be caused by an extensive condensation of graphite grains, as O'Keefe considered many years ago.

Wickramasinghe: I don't claim to have solved the formation problem. But I think that the formation of large molecules or of grains is very much more favorable near stars than in interstellar space.

Donn: Concerning the emphasis that has been given in all the calculations of the Oort-van de Hulst distributions, I think that there is no real theoretical reason for this distribution, especially when one talks about carbon grains. It is a good model in which one gets more small grains than large grains, and this is not an unreasonable assumption to work with. But I don't think one ought to feel that the precise distribution it gives is significant, and therefore, if one wants to get a different distribution he must assume that there is a series of clouds. We must certainly be free to modify this distribution in almost any way without the fear of violating a fundamental law of nature.

Donn (Communicated note (1966)): Wickramasinghe, Hudson, Stecher, and I have now reconsidered the formation of graphite in cool stars by using Tsuji's detailed calculations of molecular equilibrium. Tsuji found carbon to be highly supersaturated, a conclusion which we confirm for the regions in the atmosphere where graphite can grow to 
the required radii in sufficient time. Calculations of the nucleation rate indicate that a sufficient number of grains will form to account for the interstellar extinction. These will be ejected by radiation pressure and carry molecules along with them. Free molecules and molecules adsorbed on grains may affect the optical properties of grains and Johnson's hypothesis of the origin of diffuse bands. Graphite opacity also seriously affects the structure of the atmosphere. 